\title{
Convergence and adiabatic elimination for a driven dissipative quantum harmonic oscillator*
}

\author{
R. Azouit ${ }^{1,3}$, A. Sarlette ${ }^{2,3}$, P. Rouchon ${ }^{1,3}$
}

\begin{abstract}
We prove that a harmonic oscillator driven by Lindblad dynamics where the typical drive and loss channels are two-photon processes instead of single-photon ones, converges to a protected subspace spanned by two coherent states of opposite amplitude. We then characterize the slow dynamics induced by a perturbative single-photon loss on this protected subspace, by performing adiabatic elimination in the Lindblad dynamics.
\end{abstract}

\section{INTRODUCTION}

The harmonic oscillator is a standard quantum system. It features coherent states, equivalent of classical harmonic oscillator amplitudes, whose coherent superpositions also called "cat states" feature genuinely quantum properties with no classical equivalence. In a recent paper [8], our collaborators take advantage of this fact to propose an implementation of logical quantum bits as "cat states", with potential to realize universal quantum computation using standard technological elements to implement quantum gates. They argue that cat states present new advantages for building a quantum computer, thanks to their inherent insensitivity to part of the typical perturbations present in quantum systems. Moreover, they design an "engineered reservoir" that stabilizes a "protected subspace" of such cat states in open loop.

The contribution of the present paper is to precisely establish, from the Lindblad master equation (1), the stabilization properties of this scheme both,

- in ideal situations, by proving in theorem 1 global convergence of the infinite-dimensional nominal model towards the target "protected subspace";

- and in presence of a small but dominant decoherence source, by establishing the approximate slow dynamics as a reduced Lindblad master equation (14) on the protected subspace.

The first goal builds on a typical Lyapunov-LaSalle strategy, with additional care due to the infinite dimension. For the second goal, we resort to a separation of the quantum dynamics into fast and slow components. We

\footnotetext{
* This work was partially supported by the Projet Blanc ANR2011-BS01-017-01 EMAQS.

1 Centre Automatique et Systèmes, Mines-ParisTech, PSL Research University. 60 Bd Saint-Michel, 75006 Paris, France.

2 INRIA Paris-Rocquencourt, Domaine de Voluceau, B.P. 105, 78153 Le Chesnay Cedex, France; and Ghent University / SYSTeMS, Technologiepark 914, 9052 Zwijnaarde, Belgium.

3 QUANTIC joint project team in PSL Research University (INRIA, Mines-ParisTech, ENS Paris, Université Pierre et Marie Curie (Paris 6), CNRS).
}

then apply an adiabatic elimination of the fast system to deduce a good approximation of the dynamics on a slow manifold, with the state remaining $\epsilon$-close to the protected subspace. Studying such perturbations is standard for quantum Hamiltonian systems, where regular perturbation theory can be routinely applied [11], but the Lindbladian case with singular perturbations has attracted much less attention. In [9] and similarly [10] singular perturbations up to second order are applied to a system with $N$ ground states and eliminating relaxing excited states. In [1], [13] specific atom optics dynamics and an ancilla-mediated feedback are investigated with the standard approach of [2]. In [6] the so-called SchriefferWolff formalism is generalized to Lindblad dynamics; its basic form requires inversion of the nominal dynamics operator, which is not too practical and which we circumvent here for the derivation of the reduced slow master equation (14).

In a more general perspective, the idea of [8] is linked to the technique of protecting quantum information from typical perturbations by engineering a "protected subspace" into which the information is embedded. The paper [8] proposes a remarkably realistic and complete variation on such schemes, with explicit stabilization mechanisms onto the subspace and proposals for embodying quantum gates. Such system has been successfully implemented experimentally [14] and has shown to be a promising way towards the error correction of logical qubits in quantum information. Therefore, the precise analysis of such engineering designs is anticipated to play an important role towards tuning and quantifying the capabilities of future quantum computing hardware. In this regard the techniques proposed in the present paper, in particular some tweaks making them scalable to high-dimensional systems, might be of wider interest. We emphasize that in this paper, we focus on a realistic model of the experimental circuit and accordingly, is of practical utility.

The paper is organized as follows. Section II describes the mathematical model of the dynamics to be studied. Section III provides the global convergence proof for an idealized model, and Section IV analyzes precisely how this allows to counter the dominant external perturbation, which is single-photon loss. We pedagogically present the corresponding slow/fast perturbative argument (Section IV.B) as the translation to quantum notation of the standard dynamical systems approach, recalled in Section IV.A. Finally, simulations 
illustrate the validity of our analysis in Section V.

\section{DRIVEN DISSIPATIVE PAIRWISE PHOTON PROCESS}

The underlying space of the quantum harmonic oscillator is a Hilbert space $\mathcal{H}$ of infinite dimension spanned by the Fock states $\{|n\rangle\}_{n \in \mathbb{N}}$. Throughout this paper, we denote by $\mathcal{K}^{1}(\mathcal{H})$ the set of trace-class operators on $\mathcal{H}$, i.e. compact Hermitian operators on $\mathcal{H}$ whose eigenvalues $\left(\sigma_{k}\right)_{k \in \mathbb{N}}$ satisfy $\sum_{k \geq 0}\left|\sigma_{k}\right|<+\infty$. This $\mathcal{K}^{1}(\mathcal{H})$ equipped with the trace-norm $\operatorname{Tr}(|\cdot|)$ is a Banach space (see e.g. [12]). The quantum state space is the set of density operators, namely elements of $\mathcal{K}^{1}(\mathcal{H})$ that are positive semidefinite and of trace 1 . They are usually denoted by $\rho$. For so-called pure quantum states, $\rho$ has rank 1 and can be written $\rho=|\psi\rangle\langle\psi|$ with $|\psi\rangle$ the well-known quantum wave function; $\rho$ of higher rank allow to extend the model to open quantum systems. We denote also by $\mathcal{K}^{\mathrm{f}}(\mathcal{H})$, the subspace of $\mathcal{K}^{1}(\mathcal{H})$ whose operators' range is included in a vector space spanned by a finite number of Fock states:

$$
\mathcal{K}^{\mathrm{f}}(\mathcal{H})=\left\{\sum_{\text {finite }} f_{n, n^{\prime}}|n\rangle\left\langle n^{\prime}\right|: f_{n, n^{\prime}} \in \mathbb{C}, f_{n, n^{\prime}}=f_{n^{\prime}, n}^{*}\right\} .
$$

Isolated quantum harmonic oscillator: The annihilation operator $\boldsymbol{a}$ is defined by $\boldsymbol{a}|n\rangle=\sqrt{n}|n-1\rangle$ for any $n \geq 1$ and $\boldsymbol{a}|0\rangle=0$. Its Hermitian conjugate $\boldsymbol{a}^{\dagger}$, verifies $\boldsymbol{a}^{\dagger}|n\rangle=\sqrt{n+1}|n+1\rangle$ for any $n \geq 0$ and is called creation operator. We denote $\boldsymbol{N}=\boldsymbol{a}^{\dagger} \boldsymbol{a}$ the photon number operator, satisfying $\boldsymbol{N}|n\rangle=n|n\rangle$, for any $n \geq 0$. For any $\alpha \in \mathbb{C}$, a coherent state $|\alpha\rangle \in \mathcal{H}$ is characterized by $\boldsymbol{a}|\alpha\rangle=\alpha|\alpha\rangle$ and it writes

$$
|\alpha\rangle=e^{-\frac{|\alpha|^{2}}{2}} \sum_{n=0}^{\infty} \frac{\alpha^{n}}{\sqrt{n !}}|n\rangle .
$$

The coherent states $|\alpha\rangle$ are viewed as the quantum model for a "classical state" of complex amplitude $\alpha$. Indeed, whereas the classical undamped harmonic oscillator $\frac{d}{d t} x=-\omega p, \frac{d}{d t} p=\omega x$ with $x, p \in \mathbb{R}$ has solutions $x(t)=\alpha_{0} e^{i \omega t}$, the quantum harmonic oscillator $\frac{d}{d t}|\psi\rangle=$ $-i \omega(\boldsymbol{N}+\boldsymbol{I} / 2)|\psi\rangle$ features, up to an irrelevant phase, solutions of the form $|\psi(t)\rangle=|\alpha(t)\rangle$ i.e. a coherent state with rotating parameter $\alpha(t)=\alpha_{0} e^{i \omega t}$. As is customary, in the rest of the paper we describe the system in a frame rotating at the oscillator frequency $\omega$, for which the oscillator has stationary solutions $|\psi(t)\rangle=\left|\alpha_{0}\right\rangle$.

We call Schrödinger cat state or simply cat state the coherent superposition of two coherent states with opposite amplitudes,

$$
\left|c_{\alpha}^{ \pm}\right\rangle=\frac{|\alpha\rangle \pm|-\alpha\rangle}{\gamma^{ \pm}}
$$

where $\gamma^{ \pm}=\sqrt{2\left(1 \pm e^{-2|\alpha|^{2}}\right)}$ is a normalization factor. If $|\alpha| \gg 1$ then we have $\gamma^{+} \approx \gamma^{-} \approx \sqrt{2}$.
Open quantum harmonic oscillator : We consider the quantum harmonic oscillator interacting with its environment as described in [8]. An external coherent driving field of amplitude $u$ (assumed here real and strictly positive without loss of generality) is applied such that the oscillator can only exchange photons in pairs. Furthermore, the quantum system is engineered such that the main dissipative process is similarly a pairwise photon loss with rate $\kappa>0$. A single photon loss process is also present due to physical constraints, but it can be made much less frequent. Hence the latter's contribution appears with a small coefficient $0<\epsilon \ll \kappa$ in the following Lindblad master equation which governs the system dynamics:

$$
\frac{d}{d t} \rho=u\left[\left(\boldsymbol{a}^{\dagger}\right)^{2}-\boldsymbol{a}^{2}, \rho\right]+\kappa \mathfrak{L}_{\boldsymbol{a}^{2}}(\rho)+\epsilon \mathfrak{L}_{\boldsymbol{a}}(\rho)
$$

where $[\cdot, \cdot]$ stands for the commutator and where, for any linear operator $\boldsymbol{A}$ on $\mathcal{H}$, the super-operator $\mathfrak{L}_{\boldsymbol{A}}$ is given by

$$
\mathfrak{L}_{\boldsymbol{A}}(\rho)=\boldsymbol{A} \rho \boldsymbol{A}^{\dagger}-\left(\boldsymbol{A}^{\dagger} \boldsymbol{A} \rho+\rho \boldsymbol{A}^{\dagger} \boldsymbol{A}\right) / 2 .
$$

Noting $\alpha=2 u / \kappa$ and $\boldsymbol{L}=\boldsymbol{a}^{2}-\alpha^{2}$ one can reformulate the previous equation as :

$$
\frac{d}{d t} \rho=\kappa \mathfrak{L}_{\boldsymbol{L}}(\rho)+\epsilon \mathfrak{L}_{\boldsymbol{a}}(\rho)
$$

It is shown in [8] that, for $\epsilon=0$, the two-dimensional Hilbert space

$$
\mathcal{H}_{\alpha}=\operatorname{span}\{|\alpha\rangle,|-\alpha\rangle\}
$$

is a quantum "decoherence-free subspace", i.e. it is composed of invariant states: for $\epsilon=0$, any density operator $\bar{\rho}$ with support included in $\mathcal{H}_{\alpha}$ is a steady state, i.e., $\mathfrak{L}_{\boldsymbol{L}}(\bar{\rho})=0$.

We will not investigate here the well-posedness of (1) and the associated strongly continuous semigroup of linear contractions on $\mathcal{K}^{1}(\mathcal{H})$. Such issues could be investigated via theorem 3.1 of [3] ensuring the existence of minimal solutions since for any $\alpha, \kappa, \epsilon>0$, the operator $-\kappa \boldsymbol{L}^{\dagger} \boldsymbol{L}-\epsilon \boldsymbol{a}^{\dagger} \boldsymbol{a}$ is the infinitesimal generator of a strongly continuous one parameter contraction semigroup on $\mathcal{H}$. This means that, in the sequel, we will always assume that the Cauchy problem (1) with an initial condition $\rho_{0} \in \mathcal{K}^{1}(\mathcal{H})$, positive semidefinite and of trace one, admits a solution in $\mathcal{K}^{1}(\mathcal{H})$ defined for any $t>0$. Moreover, this minimal solution will remain positive semidefinite. We will investigate here the time asymptotic regime for $\epsilon=0$ and then for $0<\epsilon \ll \kappa$.

\section{Convergence of (1) FOR $\epsilon=0$}

Lemma 1: For any quantum state $\rho$ in $\mathcal{K}^{\mathrm{f}}(\mathcal{H})$ we have $\operatorname{Tr}\left(\boldsymbol{L} \mathfrak{L}_{\boldsymbol{L}}(\rho) \boldsymbol{L}^{\dagger}\right) \leq-2 \operatorname{Tr}\left(\boldsymbol{L} \rho \boldsymbol{L}^{\dagger}\right)$.

Proof: From $\operatorname{Tr}\left(\boldsymbol{L} \mathfrak{L}_{\boldsymbol{L}}(\rho) \boldsymbol{L}^{\dagger}\right)=\operatorname{Tr}\left(\boldsymbol{L} \rho \boldsymbol{L}^{\dagger}\left[\boldsymbol{L}^{\dagger}, \boldsymbol{L}\right]\right)$ and $\left[\boldsymbol{L}^{\dagger}, \boldsymbol{L}\right]=\left[\left(\boldsymbol{a}^{\dagger}\right)^{2}, \boldsymbol{a}^{2}\right]=-4 \boldsymbol{N}-2 \boldsymbol{I}$, we have

$$
\operatorname{Tr}\left(\boldsymbol{L} \mathfrak{L}_{\boldsymbol{L}}(\rho) \boldsymbol{L}^{\dagger}\right)=-2 \operatorname{Tr}\left(\boldsymbol{L} \rho \boldsymbol{L}^{\dagger}(2 \boldsymbol{N}+\boldsymbol{I})\right) .
$$


We conclude since $\boldsymbol{N}$ and $\boldsymbol{L} \rho \boldsymbol{L}^{\dagger}$ are positive semidefinite.

Modulo arguments proper to the infinite-dimensional setting, this basically means that $V(\rho)=\operatorname{Tr}\left(\boldsymbol{L} \rho \boldsymbol{L}^{\dagger}\right)$ is an exponential Lyapunov function for the system (1) with $\epsilon=0$. We next include some further arguments that allow us to provide a convergence theorem in the infinite-dimensional setting.

Lemma 2: For any $\nu \geq 1$ there exists $\mu \geq 0$ such that, for any quantum state $\rho$ in $\mathcal{K}^{\mathrm{f}}(\mathcal{H})$ we have

$\operatorname{Tr}\left(\mathfrak{L}_{\boldsymbol{L}}(\rho) \boldsymbol{N}^{\nu}\right) \leq-\nu\left(\operatorname{Tr}\left(\rho \boldsymbol{N}^{\nu}\right)\right)^{\frac{\nu+1}{\nu}}+\mu$.

Proof: Denote by $\mathfrak{L}_{\boldsymbol{L}}^{*}$ the adjoint of $\mathfrak{L}_{\boldsymbol{L}}$, i.e. $\quad \mathfrak{L}_{\boldsymbol{L}}^{*}(\boldsymbol{A})=\boldsymbol{L}^{\dagger} \boldsymbol{A} \boldsymbol{L}-\left(\boldsymbol{L}^{\dagger} \boldsymbol{L} \boldsymbol{A}+\boldsymbol{A} \boldsymbol{L}^{\dagger} \boldsymbol{L}\right) / 2$ for any Hermitian operator $\boldsymbol{A}$ on $\mathcal{H}$. Computations relying on the identity $\boldsymbol{a} f(\boldsymbol{N})=f(\boldsymbol{N}+\boldsymbol{I}) \boldsymbol{a}$ for any function $f$, yield

$$
\begin{aligned}
\mathfrak{L}_{\boldsymbol{L}}^{*}(f(\boldsymbol{N}))= & -\boldsymbol{N}(\boldsymbol{N}-\boldsymbol{I})(f(\boldsymbol{N})-f(\boldsymbol{N}-2 \boldsymbol{I})) \\
+\frac{\alpha^{2}}{2} \boldsymbol{a}^{2}( & f(\boldsymbol{N})-f(\boldsymbol{N}-2 \boldsymbol{I})) \\
& +\frac{\alpha^{2}}{2}(f(\boldsymbol{N})-f(\boldsymbol{N}-2 \boldsymbol{I}))\left(\boldsymbol{a}^{\dagger}\right)^{2} .
\end{aligned}
$$

This can be directly plugged in $\operatorname{Tr}\left(\mathfrak{L}_{\boldsymbol{L}}(\rho) f(\boldsymbol{N})\right)=$ $\operatorname{Tr}\left(\rho \mathfrak{L}_{\boldsymbol{L}}^{*}(f(\boldsymbol{N}))\right)$.

Take $f(x)=x^{\nu}$ and define $g(x)=f(x)-f(x-2)$ for $x \geq 2 ; g(x)=f(x)$ for $2>x \geq 0 ; g(x)=f(0)$ for $x<0$. From $\boldsymbol{a}^{2} g(\boldsymbol{N})=\boldsymbol{a} \sqrt{g(\boldsymbol{N}+\boldsymbol{I})} \boldsymbol{a} \sqrt{g(\boldsymbol{N})}$ and Cauchy-Schwarz inequality

$$
\begin{aligned}
& \left|\operatorname{Tr}\left(\rho \boldsymbol{a}^{2} g(\boldsymbol{N})\right)\right| \\
& \quad=|\operatorname{Tr}((\sqrt{\rho} \boldsymbol{a} \sqrt{g(\boldsymbol{N}+\boldsymbol{I})})(\boldsymbol{a} \sqrt{g(\boldsymbol{N})} \sqrt{\rho}))| \\
& \quad \leq \sqrt{\operatorname{Tr}(\rho g(\boldsymbol{N}+2 \boldsymbol{I})(\boldsymbol{N}+\boldsymbol{I})) \operatorname{Tr}(\rho g(\boldsymbol{N}) \boldsymbol{N})} .
\end{aligned}
$$

Since $g(x) x \leq g(x+2)(x+1)$ for $x \geq 0$, we have

$$
\left|\operatorname{Tr}\left(\rho \boldsymbol{a}^{2} g(\boldsymbol{N})\right)\right| \leq \operatorname{Tr}(\rho g(\boldsymbol{N}+2 \boldsymbol{I})(\boldsymbol{N}+\boldsymbol{I})) .
$$

Thus

$$
\frac{1}{2} \operatorname{Tr}\left(\rho\left(\boldsymbol{a}^{2} g(\boldsymbol{N})+g(\boldsymbol{N})\left(\boldsymbol{a}^{\dagger}\right)^{2}\right)\right) \leq \operatorname{Tr}(\rho g(\boldsymbol{N}+2 \boldsymbol{I})(\boldsymbol{N}+\boldsymbol{I}))
$$

and we get

$$
\begin{aligned}
& \operatorname{Tr}\left(\mathfrak{L}_{\boldsymbol{L}}(\rho) f(\boldsymbol{N})\right) \\
& \leq \operatorname{Tr}\left(\rho\left(-\boldsymbol{N}(\boldsymbol{N}-\boldsymbol{I}) g(\boldsymbol{N})+\alpha^{2} g(\boldsymbol{N}+2 \boldsymbol{I})(\boldsymbol{N}+\boldsymbol{I})\right)\right) .
\end{aligned}
$$

Since $-x(x-1) g(x)+\alpha^{2} g(x+2)(x+1)$ is dominated by the term of beneficial sign $-2 \nu x^{\nu+1}$ for large $x$, we can ensure that there exists $\mu>0$ such that

$$
\operatorname{Tr}\left(\mathfrak{L}_{\boldsymbol{L}}(\rho) \boldsymbol{N}^{\nu}\right) \leq-\nu \operatorname{Tr}\left(\rho \boldsymbol{N}^{\nu+1}\right)+\mu .
$$

Since $x^{\frac{\nu+1}{\nu}}$ is convex, $\operatorname{Tr}\left(\rho \boldsymbol{N}^{\nu+1}\right) \geq\left(\operatorname{Tr}\left(\rho \boldsymbol{N}^{\nu}\right)\right)^{\frac{\nu+1}{\nu}}$ thus $-\operatorname{Tr}\left(\rho \boldsymbol{N}^{\nu+1}\right) \leq-\left(\operatorname{Tr}\left(\rho \boldsymbol{N}^{\nu}\right)\right)^{\frac{\nu+1}{\nu}}$.

Theorem 1: Consider a trajectory $[0,+\infty[\ni t \mapsto \rho(t) \in$ $\mathcal{K}^{1}(\mathcal{H})$ of the master equation (1) with $\epsilon=0, \kappa>0$ and $\alpha>0$. The following statements hold true:
1) Take $\nu \geq 1$. Then there exists $\gamma>0$ such that, for any initial quantum state $\rho(0)=\rho_{0}$ satisfying $\operatorname{Tr}\left(\rho_{0} \boldsymbol{N}^{\nu}\right)<+\infty$, we have for all $t>0$, $\operatorname{Tr}\left(\rho(t) \boldsymbol{N}^{\nu}\right) \leq \max \left(\gamma, \operatorname{Tr}\left(\rho_{0} \boldsymbol{N}^{\nu}\right)\right)$.

2) Assume that $\rho_{0} \in \mathcal{K}^{\mathrm{f}}(\mathcal{H})$. Then, there exists a quantum state $\bar{\rho}$ with support in $\mathcal{H}_{\alpha}$ such that, for any $\nu \geq 0, \lim _{t \mapsto+\infty} \operatorname{Tr}\left(\left|\boldsymbol{N}^{\frac{\nu}{2}}(\rho(t)-\bar{\rho}) \boldsymbol{N}^{\frac{\nu}{2}}\right|\right)=0$.

The limit $\bar{\rho}$ depends on $\rho_{0}$. Its value can be uniquely derived from the constraint $\operatorname{Tr}(\bar{\rho} \xi)=\operatorname{Tr}\left(\rho_{0} \xi\right)$ for four Hermitian, bounded and independent operators $\xi$ that are in the kernel of $\mathfrak{L}_{L}^{*}$. Those $\xi$ are explicitly given in [8].

Proof: The first statement is a direct consequence of Lemma 2 and of the fact that quantum states element of $\mathcal{K}^{\mathrm{f}}(\mathcal{H})$ are a dense subset of the quantum states $\sigma$ of $\mathcal{K}^{1}(\mathcal{H})$ with $\operatorname{Tr}\left(\sigma \boldsymbol{N}^{\nu}\right)$ finite. $\operatorname{Tr}\left(\rho(t) \boldsymbol{N}^{\nu}\right)$ remains bounded since

$$
\frac{d}{d t} \operatorname{Tr}\left(\rho \boldsymbol{N}^{\nu}\right)=\kappa \operatorname{Tr}\left(\mathfrak{L}_{\boldsymbol{L}}(\rho) \boldsymbol{N}^{\nu}\right) \leq-\nu \kappa \operatorname{Tr}\left(\rho \boldsymbol{N}^{\nu}\right)^{\frac{\nu+1}{\nu}}+\kappa \mu .
$$

Thus $\frac{d}{d t} \operatorname{Tr}\left(\rho \boldsymbol{N}^{\nu}\right) \leq 0$ whenever $\operatorname{Tr}\left(\rho \boldsymbol{N}^{\nu}\right) \geq \lambda=\left(\frac{\mu}{\nu}\right)^{\frac{\nu}{\nu+1}}$,

The second statement exploits the first one. We can assume $\nu \geq 2$. Take $\nu^{\prime}>\nu$. Denote by $\mathcal{K}_{\nu^{\prime}}^{1}(\mathcal{H})$ the subspace of trace-class operators $\sigma$ such that $\operatorname{Tr}\left(\left|N^{\frac{\nu^{\prime}}{2}} \sigma N^{\frac{\nu^{\prime}}{2}}\right|\right)$ is finite. The space $\mathcal{K}_{\nu^{\prime}}^{1}(\mathcal{H})$ with the norm $\|\sigma\|_{\nu^{\prime}}=$ $\operatorname{Tr}(|\sigma|)+\operatorname{Tr}\left(\left|\boldsymbol{N}^{\frac{\nu^{\prime}}{2}} \sigma \boldsymbol{N}^{\frac{\nu^{\prime}}{2}}\right|\right)$ is a Banach space. From the first statement, we know that $\rho_{0}$ being an element of $\mathcal{K}_{\nu^{\prime}}^{1}(\mathcal{H}), \rho(t)$ remains always in $\mathcal{K}_{\nu^{\prime}}^{1}(\mathcal{H})$. Since $\nu^{\prime}>\nu$, the injection of $\mathcal{K}_{\nu^{\prime}}^{1}(\mathcal{H})$ into $\mathcal{K}_{\nu}^{1}(\mathcal{H})$ is compact: $\{\rho(t) \mid t \geq 0\}$ is precompact in $\mathcal{K}_{\nu}^{1}(\mathcal{H})$. Denote by $\bar{\rho} \in \mathcal{K}_{\nu}^{1}(\mathcal{H})$ an adherent point of $\rho(t)$ for $t$ tending towards infinity. Since $\nu \geq 2, \bar{\rho}$ and $\rho$ belong to the domain of $\mathfrak{L}_{L}$. Lemma 1 implies $\operatorname{Tr}\left(\boldsymbol{L} \bar{\rho} \boldsymbol{L}^{\dagger}\right)=0$, i.e., $\bar{\rho}$ is a steady state and its support is contained in the kernel of $\boldsymbol{L}$, which coincides with $\mathcal{H}_{\alpha}$. Moreover, the semigroup associated to the Lindblad master equation is a contraction for the trace distance: for two trajectories $\rho_{1}(t)$ and $\rho_{2}(t)$, $t \mapsto \operatorname{Tr}\left(\left|\rho_{1}(t)-\rho_{2}(t)\right|\right)$ is a non-increasing function. Thus $t \mapsto \operatorname{Tr}(|\rho(t)-\bar{\rho}|)$ is non-increasing. Consequently the adherent point $\bar{\rho}$ is unique and $\rho(t)$ converges towards $\bar{\rho}$ in $\mathcal{K}_{\nu}^{1}(\mathcal{H})$.

\section{Reduced SLOW dynamics of (1)}

We have proved in the previous section that the system converges toward the decoherence free subspace $\mathcal{H}_{\alpha}$ when we neglect the photon loss channel $(\epsilon=0)$. When $0<\epsilon \ll 1$, the center manifold theorem allows us to separate the system into fast and slow dynamics. The fast dynamics makes the system globally converge to a subspace close to $\mathcal{H}_{\alpha}$. The slow dynamics approximate the behavior of "protected states" $\left|c_{\alpha}^{+}\right\rangle,\left|c_{\alpha}^{-}\right\rangle$, which are not necessarily stationary anymore for $\epsilon>0$. The present section is aimed at characterizing these dynamics to the first order in $\epsilon$.

The fast/slow dynamics reduction from nonlinear systems theory, also known as singular perturbation theory, 
is useful despite the linearity of Lindblad dynamics, because the very high dimension and often high degeneracy of open quantum systems makes matrix diagonalization impractical to apply.

For the sake of clarity, we first particularize the fast/slow dynamics reduction theory to linear systems of finite dimension with the standard notations. We propose a reduction procedure via an adapted duality viewpoint with the characterization based on (8) and (9) here below. We then apply this characterization to the quantum system (1) and show that it facilitates the computations up to first order, as compared to e.g. [6].

\section{A. Reducing a linear system to its slow dynamics}

We here review the theory of geometric singular perturbations, which was mainly developed by Fenichel in [4] and surveyed by Jones in [5], in a linear context. Consider the nominal linear system $\frac{d}{d t} x=A x$ with $x=\left(x_{1}, x_{2}\right) \in \mathbb{R}^{m} \times \mathbb{R}^{n}$ and converging globally to the $m$ dimensional subspace $\mathcal{S}=\left\{x \in \mathbb{R}^{m+n}: \mathbb{R}^{n} \ni x_{2}=0\right\}$. For simplicity, we further assume that $A x=0$ for all $x \in \mathcal{S}$. To this nominal dynamics we add an arbitrary perturbation $B x$ of order $\epsilon \ll 1$. In matrix notation, the dynamics can be written in block form which yields:

$$
\begin{aligned}
& \frac{d}{d t} x_{1}=A_{1} x_{2}+\epsilon\left(B_{1} x_{2}+B_{0} x_{1}\right) \\
& \frac{d}{d t} x_{2}=A_{2} x_{2}+\epsilon\left(B_{2} x_{2}+B_{3} x_{1}\right) .
\end{aligned}
$$

The assumption that $\mathcal{S}$ is globally exponentially stable for $\epsilon=0$ corresponds to the eigenvalues of $A_{2}$ all having strictly negative real parts, thus $A_{2}$ is invertible. Hence we can define the regular change of variables

$$
\begin{aligned}
& \tilde{x}_{1}=x_{1}-A_{1} A_{2}^{-1} x_{2} \\
& \tilde{x}_{2}=x_{2}
\end{aligned}
$$

which yields dynamics in Tikhonov normal form

$$
\begin{aligned}
\frac{d}{d t} \tilde{x}_{1}= & \epsilon\left(\left(B_{0}-A_{1} A_{2}^{-1} B_{3}\right) \tilde{x}_{1}\right. \\
& \left.\quad+\left(B_{0}+B_{1}-A_{1} A_{2}^{-1}\left(B_{2}+B_{3}\right)\right) \tilde{x}_{2}\right) \\
=: & \epsilon f\left(\tilde{x}_{1}, \tilde{x}_{2}\right) . \\
\frac{d}{d t} \tilde{x}_{2}= & A_{2} \tilde{x}_{2}+\epsilon\left(B_{3} \tilde{x}_{1}+\left(B_{2}+B_{3} A_{1} A_{2}^{-1}\right) \tilde{x}_{2}\right) \\
=: & g\left(\tilde{x}_{1}, \tilde{x}_{2}, \epsilon\right) .
\end{aligned}
$$

The Tikhonov conditions for reducing the system by singular perturbations is that the first (slow) subsystem has eigenvalues of order $\epsilon$, while the second (fast) subsystem has eigenvalues bounded away from zero for $\epsilon=0$. These conditions are satisfied above. The Tikhonov theorem then allows the following reduction.

Proposition 1: The trajectories of the full system $(4),(5)$ (with initial conditions satisfying $g=0$ ) remain $\epsilon$ close over at least a time of order $1 / \epsilon$, to the trajectories of a reduced "slow system". The latter is restricted to the "slow submanifold" defined by $g\left(\tilde{x}_{1}, \tilde{x}_{2}, 0\right)=0$; its dynamics can be parameterized by letting $\tilde{x}_{1}$ evolve according to $f\left(\tilde{x}_{1}, \tilde{x}_{2}\right)$, in which $\tilde{x}_{2}$ is replaced by the solution of $g\left(\tilde{x}_{1}, \tilde{x}_{2}, 0\right)=0$ as a function of $\tilde{x}_{1}$.
In our linear case, the slow manifold comes down to $\tilde{x}_{2}=0$ and the slow dynamics trivially reduce to the first term in (4). Also transforming back to the original coordinates, the slow manifold corresponds just to $x_{2}=0$ and the slow system dynamics are

$$
\frac{d}{d t} x_{1}=\epsilon\left(B_{0}-A_{1} A_{2}^{-1} B_{3}\right) x_{1} .
$$

The second term reflects the influence of the fast $x_{2}$ dynamics on the slow variable $x_{1}$ : by blindly setting $x_{2}=0$ in the original system (2) and neglecting its second line, we would miss this term and get an incorrect approximation.

Computing the corrective term $A_{1} A_{2}^{-1} B_{3}$ by explicit inversion of $A_{2}$ can be a tedious task when the fast subsystem has a large dimension (in our quantum case, $x_{2}$ would rigorously be of infinite dimension). However, the computations can be facilitated via the following dual viewpoint, using the first integrals of the system with $\epsilon=0$.

Consider a linear functional $p^{T}=\left(p_{1}^{T}, p_{2}^{T}\right) \in \mathbb{R}^{* m+n}$ which is conserved by $\dot{x}=A x$, i.e. satisfying $\dot{p}=A^{T} p=0$ or equivalently $p_{1}^{T} A_{1} x_{2}+p_{2}^{T} A_{2} x_{2}=0$ for all $x_{2} \in \mathbb{R}^{n}$. (The notation $\cdot^{T}$ denotes matrix transpose.) Again using invertibility of $A_{2}$, we see that $p^{T}$ actually satisfies

$$
p_{1}^{T} A_{1} A_{2}^{-1} y+p_{2}^{T} y=0 \quad \forall y \in \mathbb{R}^{n} .
$$

Hence knowing $m$ linearly independent functionals $\left\{p^{T}(k)\right\}_{k=1, \ldots, m}$ satisfying (7) is sufficient to fully characterize the corrective term in (6): we have

$$
\frac{d}{d t} x_{1}=\epsilon\left(B_{0}+Q\right) x_{1}
$$

with $Q$ defined by the set of linear equations:

$$
p_{1}^{T}(k) Q=p_{2}^{T}(k) B_{3}, k=1,2, \ldots, m .
$$

This system can be easier to solve than computing $A_{1} A_{2}^{-1} B_{3}$ explicitly, as we now illustrate with our application.

\section{B. Quantum system (1) with $0<\epsilon \ll \kappa$}

We now apply the same procedure to our quantum system. The nominal $\dot{x}=A x$ corresponds to $\dot{\rho}=\mathfrak{L}_{\boldsymbol{L}}(\rho)$. We have shown in Section III that:

- $\mathcal{S}$ corresponds to a four-dimensional real subspace of Hermitian operators spanned by $\left|c_{\alpha}^{+}\right\rangle\left\langle c_{\alpha}^{+}|,| c_{\alpha}^{-}\right\rangle\left\langle c_{\alpha}^{-}\right|$, $\left|c_{\alpha}^{+}\right\rangle\left\langle c_{\alpha}^{-}|+| c_{\alpha}^{-}\right\rangle\left\langle c_{\alpha}^{+}\right|$, and $i\left(\left|c_{\alpha}^{+}\right\rangle\left\langle c_{\alpha}^{-}|-| c_{\alpha}^{-}\right\rangle\left\langle c_{\alpha}^{+}\right|\right)$. These correspond to the $m=4$ coordinates of $x_{1}$.

- The subspace $\mathcal{S}$ is globally asymptotically stable under $\dot{\rho}=\mathfrak{L}_{\boldsymbol{L}}(\rho)$. This corresponds to the invertibility condition on $A_{2}$ independently of $\epsilon$. For any finite-dimensional approximation, convergence of the linear system would automatically be exponential and thus the system in proper reformulation would satisfy the Tikhonov conditions.

We therefore introduce the projector

$$
\boldsymbol{P}_{c}=\left|c_{\alpha}^{+}\right\rangle\left\langle c_{\alpha}^{+}|+| c_{\alpha}^{-}\right\rangle\left\langle c_{\alpha}^{-}\right|
$$


such that

$$
\rho_{s}=\boldsymbol{P}_{c} \rho \boldsymbol{P}_{c}
$$

corresponds to the "slow" $x_{1}$ space of the previous section. The projection onto " $x_{2}$ space" is given by

$$
\rho_{f}=\rho-\boldsymbol{P}_{c} \rho \boldsymbol{P}_{c}
$$

Our goal is to compute the dynamics of $\rho_{s}$ parameterizing the slow system, which is the equivalent of (6). For this we take advantage of the dual formulation $(8),(9)$. The following procedure can in principle be applied to any perturbative dynamics, we here focus on $\mathfrak{L}_{\boldsymbol{a}}$ as a physically relevant case. Single photon loss is indeed the most prominent disturbance encountered, expressing that the system slowly loses energy by radiative thermal interaction with an ultra-cold environment.

The perturbative dynamics on the slow manifold features a first component, corresponding to $B_{0}$, which is obtained simply by projection onto the slow manifold. The identities $\boldsymbol{a}\left|c^{ \pm}\right\rangle=\alpha \frac{\gamma^{\mp}}{\gamma^{ \pm}}\left|c^{\mp}\right\rangle$ quickly yield its explicit expression:

$$
\begin{aligned}
\boldsymbol{P}_{c} \mathfrak{L}_{\boldsymbol{a}}\left(\rho_{s}\right) \boldsymbol{P}_{c} & =\alpha^{2} \mathfrak{L}_{\boldsymbol{X}}\left(\rho_{s}\right) \\
\text { where } \boldsymbol{X} & =\frac{\gamma_{+}}{\gamma_{-}}\left|c^{+}\right\rangle\left\langle c^{-}\left|+\frac{\gamma_{-}}{\gamma_{+}}\right| c^{-}\right\rangle\left\langle c^{+}\right| .
\end{aligned}
$$

To compute the corrective term by duality, using the equivalent of (8),(9), we need to identify $m=4$ conserved functionals of the system, which are the fixed points of the dual nominal dynamics $\frac{d}{d t} \xi=\mathfrak{L}_{\boldsymbol{L}}^{*}(\xi)$. Fortunately, those invariants are known for the particular operator $\boldsymbol{L}$ :

- One easily checks that $\xi^{a}=\boldsymbol{I}$ the identity operator is in the kernel of any $\mathfrak{L}_{L}^{*}$.

- The parity operator $\xi^{b}=(-1)^{\boldsymbol{a}^{\dagger} \boldsymbol{a}}$ is in the kernel of $\mathfrak{L}_{L}^{*}$ because photons are exchanged by pairs.

- The appendix of [8] gives two more operators $\xi^{c}$ and $\xi^{d}$ in terms of Bessel functions; one checks that they are linearly independent for finite $\alpha$.

We will also use the following key property of the conserved quantities, which is specific to the structure of quantum Lindblad dynamics.

Lemma 3: Any Hermitian operator $\xi$ in $\operatorname{ker}\left(\mathfrak{L}_{L}^{*}\right)$ commutes with $\boldsymbol{P}_{c}$ the orthogonal projector onto $\mathcal{H}_{\alpha}$.

Proof: From $2 \boldsymbol{L}^{\dagger} \xi \boldsymbol{L}=\boldsymbol{L}^{\dagger} \boldsymbol{L} \xi+\xi \boldsymbol{L}^{\dagger} \boldsymbol{L}$ and $\boldsymbol{L} \boldsymbol{P}_{c}=$ $0=\boldsymbol{P}_{c} \boldsymbol{L}^{\dagger}$ we have $\boldsymbol{L}^{\dagger} \boldsymbol{L} \xi \boldsymbol{P}_{c}=0=\boldsymbol{P}_{c} \xi \boldsymbol{L}^{\dagger} \boldsymbol{L}$. Thus the Hermitian operator $\boldsymbol{A}=\boldsymbol{P}_{c} \xi+\xi \boldsymbol{P}_{c}$ satisfies $\boldsymbol{L} \boldsymbol{A} \boldsymbol{L}^{\dagger}=$ $\left(\boldsymbol{L}^{\dagger} \boldsymbol{L} \boldsymbol{A}+\boldsymbol{A} \boldsymbol{L}^{\dagger} \boldsymbol{L}\right) / 2(=0)$, i.e. it belongs to the kernel of $\mathfrak{L}_{\boldsymbol{L}}$. The support of $\boldsymbol{A}$ is thus included in $\mathcal{H}_{\alpha}$ and thus $\left[\boldsymbol{P}_{c}, \boldsymbol{A}\right]=0$. This implies that $\boldsymbol{P}_{c} \xi=\xi \boldsymbol{P}_{c}$.

The equivalent of the $B_{3}$ term of the linear perturbation is given by $\mathfrak{L}_{\boldsymbol{a}}\left(\rho_{s}\right)-\boldsymbol{P}_{c} \mathfrak{L}_{\boldsymbol{a}}\left(\rho_{s}\right) \boldsymbol{P}_{c}$. Then the equivalent of equation (9) characterizes the Hermitian operator $\boldsymbol{Q}$ with support on $\mathcal{H}_{\alpha}$ as follows:

$$
\begin{aligned}
& \operatorname{Tr}\left(\boldsymbol{P}_{c} \xi^{\nu} \boldsymbol{P}_{c} \boldsymbol{Q}\right) \\
&= \operatorname{Tr}\left(\left(\xi^{\nu}-\boldsymbol{P}_{c} \xi^{\nu} \boldsymbol{P}_{c}\right)\left(\mathfrak{L}_{a}\left(\rho_{s}\right)-\boldsymbol{P}_{c} \mathfrak{L}_{a}\left(\rho_{s}\right) \boldsymbol{P}_{c}\right)\right) \\
& \quad=\operatorname{Tr}\left(\xi^{\nu}\left(\mathfrak{L}_{a}\left(\rho_{s}\right)-\boldsymbol{P}_{c} \mathfrak{L}_{a}\left(\rho_{s}\right) \boldsymbol{P}_{c}\right)\right) \\
&=\frac{1}{2} \operatorname{Tr}\left(\xi^{\nu}\left(\left(\boldsymbol{P}_{c}-I\right) a^{\dagger} a \rho_{s}+\rho_{s} a^{\dagger} a\left(\boldsymbol{P}_{c}-I\right)\right)\right) \\
&=\frac{1}{2} \operatorname{Tr}\left(\xi^{\nu}\left(a^{\dagger} a \rho_{s}\left(\boldsymbol{P}_{c}-I\right)+\left(\boldsymbol{P}_{c}-I\right) \rho_{s} a^{\dagger} a\right)\right)
\end{aligned}
$$

for any $\nu=a, b, c, d$. From the first to the second line above we just express (9); from the second to the third line, $\boldsymbol{P}_{c} \xi^{\nu} \boldsymbol{P}_{c}$ is readily dropped since $\boldsymbol{P}_{c}$ is a projector. Towards the next line we have to write out $\mathfrak{L}_{\boldsymbol{a}}$ and see that $\boldsymbol{P}_{c} \boldsymbol{a} \rho_{s} \boldsymbol{a}^{\dagger} \boldsymbol{P}_{c}=\boldsymbol{a} \rho_{s} \boldsymbol{a}^{\dagger}$ for the particular perturbation operator $\boldsymbol{a}$. The last two lines follow by using Lemma 3 and $\boldsymbol{P}_{c} \rho_{s}=\rho_{s}$. As a conclusion, we get that the corrective term is $\boldsymbol{Q}=0$ for our particular case. We can summarize these computations as follows. We do not call this a theorem, only because we did not completely treat the Tikhonov conditions for an infinite-dimensional system. It appears however that for any finite-dimensional approximation, this should characterize the dynamics accurately.

Proposition 2: The trajectories of system (1) with initial conditions having support in $\mathcal{H}_{\alpha}$ remain $\epsilon$-close over at least a time of order $1 / \epsilon$, to the trajectories of the "slow system" parameterized by $\rho_{s}$ which is a linear combination of $\left|c_{\alpha}^{+}\right\rangle\left\langle c_{\alpha}^{+}|,| c_{\alpha}^{-}\right\rangle\left\langle c_{\alpha}^{-}|,| c_{\alpha}^{+}\right\rangle\left\langle c_{\alpha}^{-}|+| c_{\alpha}^{-}\right\rangle\left\langle c_{\alpha}^{+}\right|$, and $i\left(\left|c_{\alpha}^{+}\right\rangle\left\langle c_{\alpha}^{-}|-| c_{\alpha}^{-}\right\rangle\left\langle c_{\alpha}^{+}\right|\right)$. The slow system follows the Lindblad dynamics:

$$
\begin{aligned}
\frac{d}{d t} \rho_{s} & =\epsilon \alpha^{2} \mathfrak{L}_{\boldsymbol{X}}\left(\rho_{s}\right) \\
\text { where } \boldsymbol{X} & =\frac{\gamma_{+}}{\gamma_{-}}\left|c^{+}\right\rangle\left\langle c^{-}\left|+\frac{\gamma_{-}}{\gamma_{+}}\right| c^{-}\right\rangle\left\langle c^{+}\right| .
\end{aligned}
$$

In applications, considering $\left|c_{\alpha}^{+}\right\rangle$and $\left|c_{\alpha}^{-}\right\rangle$as canonical states $|0\rangle,|1\rangle$ of a logical qubit [8], the operator $X$ corresponds to a bit-flip in the limit $\gamma_{+} / \gamma_{-} \rightarrow 1$ of large coherent amplitude $\alpha$; and to a decoherence to the vacuum $|0\rangle$ in the limit of Fock states $\left|c_{\alpha}^{+}\right\rangle=|n=0\rangle$, $\left|c_{\alpha}^{-}\right\rangle=|n=1\rangle$ when $\alpha=0$. For all other cases, the qubit dynamics $(14),(15)$ corresponds on the canonical Bloch sphere to:

$$
\begin{aligned}
\frac{d}{d t} x & =-\alpha^{2} \frac{\left(\gamma_{+}^{2}-\gamma_{-}^{2}\right)^{2}}{2 \gamma_{+}^{2} \gamma_{-}^{2}} x \\
\frac{d}{d t} y & =-\alpha^{2} \frac{\left(\gamma_{+}^{2}+\gamma_{-}^{2}\right)^{2}}{2 \gamma_{+}^{2} \gamma_{-}^{2}} y \\
\frac{d}{d t} z & =-\alpha^{2} \frac{\gamma_{+}^{4}+\gamma_{-}^{4}}{\gamma_{+}^{2} \gamma_{-}^{2}}\left(z-\frac{\gamma_{+}^{4}-\gamma_{-}^{4}}{\gamma_{+}^{4}+\gamma_{-}^{4}}\right) .
\end{aligned}
$$

This converges to $x=0$ (slowly for $\frac{\gamma_{+}}{\gamma_{-}} \simeq 1$ ), $y=0$, and $z=\frac{\gamma_{+}^{4}-\gamma_{-}^{4}}{\gamma_{+}^{4}+\gamma_{-}^{4}}\left(\right.$ which is $\simeq 0$ for $\left.\frac{\gamma_{+}}{\gamma_{-}} \simeq 1\right)$.

\section{Numerical Simulations}

To validate our approach and conclusions, the following numerical simulations compare trajectories of the 
complete system (1) to those obtained with the reduced approximate system (14),(15). We use a numerical scheme which preserves the positiveness for the Lindblad equation, similar to the one used in [7]. The following numerical values are fixed: $\kappa=1, u=1 / 2, \epsilon=0.01$ and an integration time-step of $10^{-3}$. For the resulting value $\alpha=1$, we have $|\langle n \mid \alpha\rangle|^{2}<1 / n$ ! while $\sum_{n=0}^{+\infty}|\langle n \mid \alpha\rangle|^{2}=$ 1. Consequently, one easily checks that truncating the infinite-dimensional Hilbert space to a numerical system space spanned by $\{|1\rangle,|2\rangle, \ldots|40\rangle\}$ in the Fock basis covers all but a numerically negligible part of the state. We denote by $\rho$ the density matrix of the resulting system. It is worth stressing that the complete system is represented by an $n_{\max } \times n_{\max }$ matrix while the reduced system is represented by a $2 \times 2$ matrix (on the basis $\left.\left|c_{\alpha}^{+}\right\rangle,\left|c_{\alpha}^{-}\right\rangle\right)$. Thus the computation is much faster on the second one.

We first take as initial condition the vacuum state, $\rho_{0}=|0\rangle\langle 0|$. The state of the reduced system, $\rho_{s}$ is then initialized at $\left|c_{\alpha}^{+}\right\rangle\left\langle c_{\alpha}^{+}\right|$because both $\rho_{0}$ and $\left|c_{\alpha}^{+}\right\rangle\left\langle c_{\alpha}^{+}\right|$are +1 eigenstates of the parity operator $\xi^{b}=(-1)^{a^{\dagger} a}$, which is a conserved quantity (see Section IV-B and [8]). To compare the trajectories of (1) initialized at $|0\rangle\langle 0|$ and of (14) initialized at $\left|c_{\alpha}^{+}\right\rangle\left\langle c_{\alpha}^{+}\right|$, we show in figure 1 the expectation values $\operatorname{Tr}\left(\rho \sigma_{z}\right)$ and $\operatorname{Tr}\left(\rho_{s} \sigma_{z}\right)$ of the operator $\sigma_{z}=\left|c_{\alpha}^{+}\right\rangle\left\langle c_{\alpha}^{+}|-| c_{\alpha}^{-}\right\rangle\left\langle c_{\alpha}^{-}\right|$, commonly denoted $\left\langle\sigma_{z}\right\rangle$. After a transitional regime of typical duration $1 / \kappa$, one can see a strong similarity between $\operatorname{Tr}\left(\rho \sigma_{z}\right)$ and $\operatorname{Tr}\left(\rho_{s} \sigma_{z}\right)$ up to a constant offset. The value of this offset is of order $\epsilon$. Furthermore, we plot the fidelity $F\left(\rho, \rho_{s}\right)=$ $\operatorname{tr}\left(\sqrt{\sqrt{\rho_{s}} \rho \sqrt{\rho_{s}}}\right)$ between $\rho_{s}$ and $\rho$. For better readability, figure 2 shows the logarithm of 1 minus the fidelity, i.e. of its deviation from the ideal value 1 . This deviation quickly converges to an order $10^{-4}$, corresponding to $\epsilon^{2}$ as expected. It then further decreases, incidentally, as both systems converge towards the unique equilibrium of the slow dynamics.

To emphasize the influence of $\gamma^{+}$and $\gamma^{-}$in (15), we add a simulation with the same parameters, but with the complete and reduced system having an identical initial condition:

$$
\tilde{\rho}_{0}=\frac{1}{2}\left(\left|c_{\alpha}^{+}\right\rangle+\left|c_{\alpha}^{-}\right\rangle\right)\left(\left\langle c_{\alpha}^{+}\right|+\left\langle c_{\alpha}^{-}\right|\right)
$$

Figure 3 shows that the expectation value of $\sigma_{x}=$ $\left|c_{\alpha}^{+}\right\rangle\left\langle c_{\alpha}^{-}|+| c_{\alpha}^{-}\right\rangle\left\langle c_{\alpha}^{+}\right|$slowly decreases over time, as expected from bit-flip dynamics. The slope of this decrease is approximated to $\sim 4 \%$ accuracy by the reduced dynamics. Moreover, figure 4 establishes that $\left\langle\sigma_{z}\right\rangle$ does not remain zero. This is due to the fact that, with $\gamma^{+}>\gamma^{-}$, equation (14) "promotes" the population of $\left|c_{\alpha}^{+}\right\rangle\left\langle c_{\alpha}^{+}\right|$over the population of $\left|c_{\alpha}^{-}\right\rangle\left\langle c_{\alpha}^{-}\right|$, unlike a pure bit-flip.

The simulations thus confirm the validity of our approximation of the complete model by the reduced one.

\section{CONCLUSIONS}

We have rigorously proved convergence of a harmonic oscillator Lindblad dynamics with two-photon

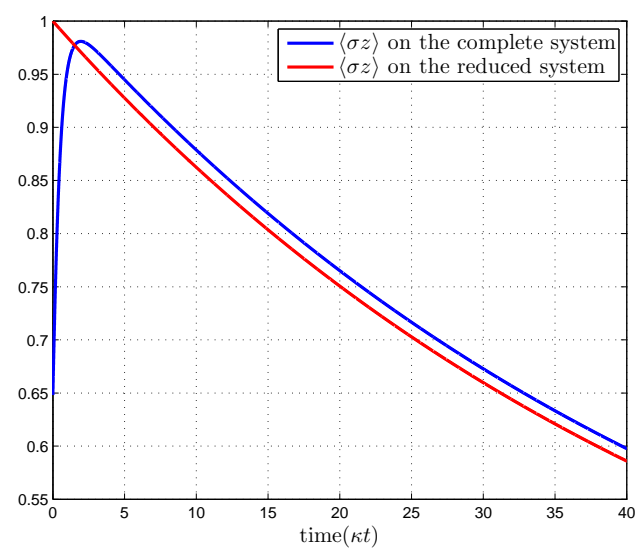

Fig. 1. Comparison of $\operatorname{Tr}\left(\sigma_{z} \rho\right)$ and $\operatorname{Tr}\left(\sigma_{z} \rho_{s}\right)$ for $\rho$ solution of the complete system (1) with $\epsilon=\frac{1}{100}, \alpha=1$ and vacuum initial condition (truncation up to 40 photons), and for $\rho_{s}$ solution of the reduced system (14) with initial condition $\left|c_{\alpha}^{+}\right\rangle\left\langle c_{\alpha}^{+}\right|$.

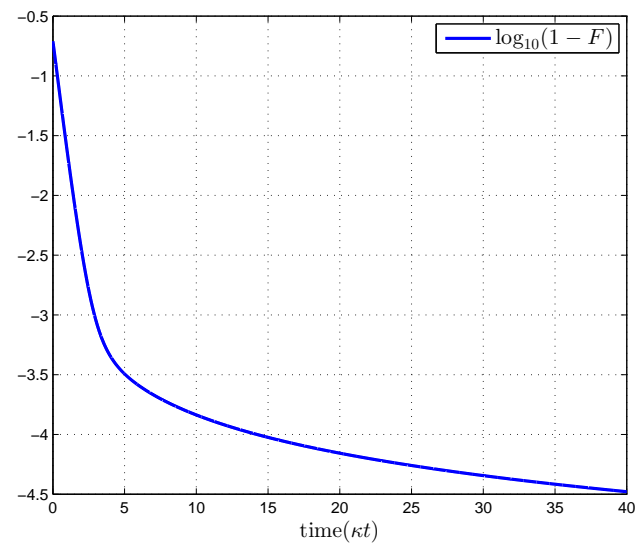

Fig. 2. $\log _{10}(1-F)$ where $F$ is the fidelity between $\rho_{s}$ and $\rho$ from the simulations of figure 1 .

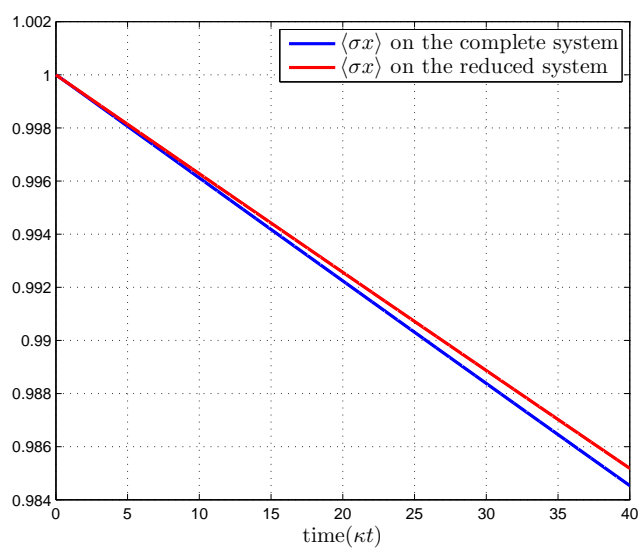

Fig. 3. Comparison of $\operatorname{Tr}\left(\sigma_{x} \rho\right)$ and $\operatorname{Tr}\left(\sigma_{x} \rho_{s}\right)$ for $\rho$ solution of the complete system (1) with $\epsilon=\frac{1}{100}, \alpha=1$ (truncation up to 40 photons), and for $\rho_{s}$ solution of the reduced system (14), with the same initial condition $\rho(0)=\rho_{s}(0)=\frac{1}{2}\left(\left|c_{\alpha}^{+}\right\rangle+\left|c_{\alpha}^{-}\right\rangle\right)\left(\left\langle c_{\alpha}^{+}\right|+\left\langle c_{\alpha}^{-}\right|\right)$. Note the vertical scale. 


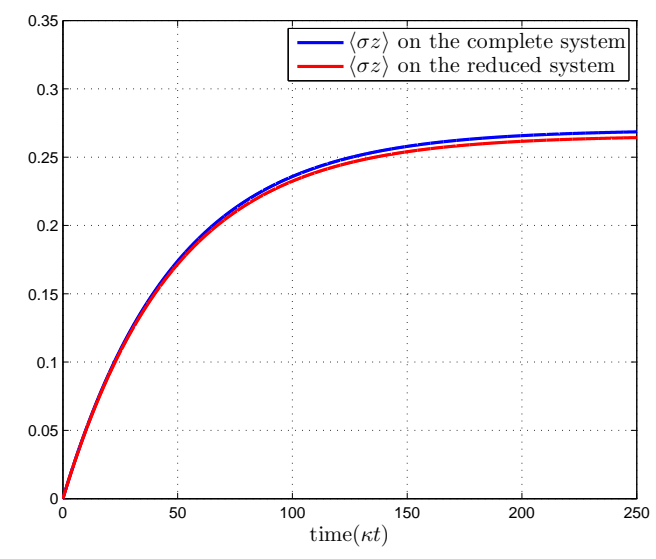

Fig. 4. Comparison of $\operatorname{Tr}\left(\sigma_{z} \rho\right)$ and $\operatorname{Tr}\left(\sigma_{z} \rho_{s}\right)$ where $\rho$ and $\rho_{s}$ correspond to simulations of figure 3 .

exchanges, to a protected subspace. We have also established the approximate slow dynamics on this protected subspace when a typical perturbation is added, and illustrated its validity in simulations. The methods used for this particular example are applicable to general Lindblad dynamics.

In particular, the approach addresses the important practical question of evaluating the remaining slow dynamics in quantum systems with (engineered) protected subspaces. Extension of the present model to $k$-photon processes $\boldsymbol{a}^{k}$ with $k>2$ can be addressed in the same way. The fact that the slow variable still follows a Lindbladian master equation may not be surprising but remains to be proved in the general case. The fact that the dynamics reduces to the orthogonal projection of the Lindbladian onto the protected subspace (i.e. $B_{0}$ without any correction due to $B_{3}$, in the terms of Section IV-A) for the particular case examined here would be in agreement with the physicists' "quantum Zeno" viewpoint. However, under which formulation this viewpoint should be applied in the general case also remains to be rigorously characterized.

\section{ACKNOWLEDGMENT}

The authors thank Mazyar Mirrahimi for many useful discussions.

\section{REFERENCES}

[1] D.J. Atkins, H. Wiseman, and P. Warszawski. Approximate master equations for atom optics. Physical Review A, 67(2):023802, 2003.

[2] H. . Carmichael. An Open Systems Approach to Quantum Optics. Springer-Verlag, 1993.

[3] E.B. Davies. Quantum dynamical semigroups and the neutron diffusion equation. Reports on Mathematical Physics, 11(2):169-188, April 1977.

[4] N. Fenichel. Geometric singular perturbation theory for ordinary differential equations. J. Diff. Equations, 31:53-98, 1979.

[5] C.K.R.T. Jones. Geometric singular perturbation theory. In Russell Johnson, editor, Lecture Notes in Mathematics, volume 1609, pages 44-118-. Springer Berlin Heidelberg, 1995.

[6] E. M. Kessler. Generalized Schrieffer-Wolff formalism for dissipative systems. Phys. Rev. A, 86(1):012126-, July 2012.
[7] C. Le Bris and P. Rouchon. Low-rank numerical approximations for high-dimensional lindblad equations. Phys. Rev. A, 87(2):022125-, February 2013.

[8] M. Mirrahimi, Z. Leghtas , V.V. Albert, S. Touzard, R.J.. Schoelkopf, L. Jiang, and M.H. Devoret. Dynamically protected cat-qubits: a new paradigm for universal quantum computation. New Journal of Physics, 16:045014, 2014.

[9] M. Mirrahimi and P. Rouchon. Singular perturbations and Lindblad-Kossakowski differential equations. IEEE Trans. Automatic Control, 54(6):1325-1329, 2009.

[10] F. Reiter and A.S. Sørensen. Effective operator formalism for open quantum systems. Phys. Rev. A, 85(3):032111-, March 2012.

[11] J.J. Sakurai and J. Napolitano. Modern quantum mechanics. Addison-Wesley, 2011.

[12] V.E. Tarasov. Quantum Mechanics of Non-Hamiltonian and Dissipative Systems. Elsevier, 2008.

[13] P. Warszawski and H. Wiseman. Adiabatic elimination in compound quantum systems with feedback. Physical Review A, 63(1):013803, 2000.

[14] Z. Leghtas, S. Touzard, I.M. Pop, A. Kou, B. Vlastakis, A. Petrenko, K.M. Sliwa, A. Narla, S. Shankar, M.J. Hatridge, M. Reagor, L. Frunzio, R.J. Schoelkopf, M. Mirrahimi and M.H. Devoret. Confining the state of light to a quantum manifold by engineered two-photon loss. Science, 347(6224):853-857, 2015. 